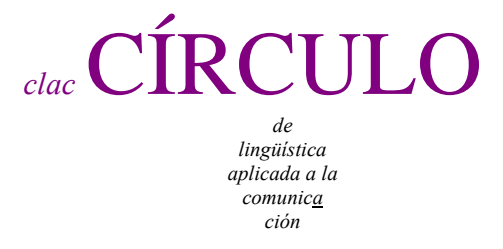

$55 / 2013$

\title{
A PROPÓSITO DE CIERTAS FÓRMULAS TEMATIZADORAS, SUS PROPIEDADES GRAMATICALES Y SU RENDIMIENTO DISCURSIVO
}

\author{
Taresa Fernández Lorences \\ Universidad de Oviedo \\ lorencestaresa en uniovi. s
}

Resumen

Algunas expresiones lingüísticas están especializadas en presentar el tema del que se habla o en introducir un cambio de tema en el discurso. Se seleccionan aquí tres de ellas, en relación con, por cierto y a propósito (de), con el fin de determinar sus diferencias en el plano gramatical (nivel de fijación de sus componentes y relación sintáctica con la oración) y su rendimiento en el discurso como elementos cohesionadores de la estructura informativa del texto.

La primera (en relación con) representa un conjunto variado de unidades (acerca de, en cuanto a, en lo relativo a, en lo que respecta $a$, por lo que toca a, etc.) que sistemáticamente aparecen para introducir un sustantivo o unidad sustantivada que se presenta como tema de la oración, y que resulta imprescindible para la viabilidad de la construcción. En contraste con estas, por cierto nunca va acompañada de ninguna expresión referencial, y su papel discursivo consiste en advertir al interlocutor de que se va a producir una digresión. Entre ambos extremos de la escala se encuentra a propósito (de). Esta última presenta un comportamiento mixto, pues en ocasiones puede ir seguida de un sustantivo al que marca como tema, mientras que en otros casos aparece de forma escueta y equivale a por cierto.

Palabras clave: tema, predicado, fórmulas tematizadoras, gramática, discurso.

Fernández Lorences, Taresa. 2013.

A propósito de ciertas fórmulas tematizadoras, sus propiedades gramaticales y su rendimiento discursivo.

http://www.ucm.es/info/circulo/no55/lorences.pdf

Círculo de Lingüística Aplicada a la Comunicación 55, 40-60.

http://www.ucm.es/info/circulo/no55/duque.pdf http://revistas.ucm.es/index.php/CLAC DOI http://dx.doi.org/10.5209/rev_CLAC.2013.v55.43265

(C)2013 Taresa Fernández Lorences

Círculo de Lingüística Aplicada a la Comunicación (clac)

Universidad Complutense de Madrid. ISSN 1576-4737. http://www.ucm.es/info/circulo 


\begin{abstract}
Concerning certain topic markers, their grammatical properties and their performance in discourse

Some linguistic expressions are specialized in presenting the topic of conversation or in introducing a change in the topic of discourse. Here, three such expressions have been selected: en relación con, por cierto and a propósito (de), with the goal of determining their differences on a grammatical level (establishing their components and their syntactic relationship within a sentence) as well as their performance in a dialogue as cohesive elements of the text's informative structure.

The first expression (en relación con) represents a varied set of components (acerca de, en cuanto a, en lo relativo a, en lo que respecta a, por lo que toca a, etc.) that systematically appear in order to introduce a noun or a nominalized unit that is presented as the subject of the sentence, and that is consequently essential to the construction's viability. In contrast, por cierto never is accompanied by a referential expression, and its discursive role consists of informing the interlocutor that a digression is about to occur. On the scale between these extremes, we find a propósito (de). This last expression presents a mixed behavior. On some occasions, it can be followed by a noun that it marks as the subject, while on others it appears in a reduced form and equates to por cierto.
\end{abstract}

Key words: topic, predicate, topic markers, grammar, syntax.

Índice

1. Introducción 42

2. La fórmula en relación con ( en relación a con relación a) 44

3. La locución por cierto 46

4. La fórmula a propósito 54

Bibliografía 58 


\section{Introducción}

Algunas expresiones lingüísticas cumplen la función de presentar un tema o de anunciar un cambio de tema en el tejido discursivo. Con el fin de analizar sus similitudes y diferencias, hemos seleccionado tres de ellas (en relación con, por cierto y a propósito) por considerarlas representativas de distintos comportamientos, tanto en el plano gramatical como por los valores que aportan a la estructura informativa del discurso.

En conjunto, constituyen fórmulas que sirven a la tematización, entendida aquí como el procedimiento mediante el cual un sustantivo o grupo de categoría sustantiva se destaca por medios gramaticales (morfosintácticos, entonativos o léxicos) llevándolo al comienzo (o, en ciertos casos, al final) de la oración y marcándolo como tema de esta, con la que se relaciona desde una posición extrapredicativa. En este sentido, las unidades tematizadas comparten con otros incisos el hecho de sustraerse a la cobertura de los morfemas extensos del verbo, pues ya no establecen con el núcleo verbal una relación de dependencia directa en alguna de las funciones oracionales (Fernández Lorences 2010: 77-78).

La relación sintáctica que se establece entre la unidad tematizada (que podemos denominar provisionalmente tema lingüístico) y la oración no presenta cambios sustanciales cuando el tema está codificado en un mero sustantivo actualizado (1) o cuando va precedido de una de las fórmulas introductoras de tema (2):

(1) Mi hermana, mis padres siempre se preocupan por ella.

(2) En cuanto a mi hermana, mis padres siempre se preocupan por ella.

Los incisos temáticos como el recogido en (1) aparecen frecuentemente en los usos orales y coloquiales, pero desde el punto de vista formal siempre admiten la anteposición de una locución del tipo en cuanto $a$. La finalidad de estas locuciones no es otra que aportar indicios léxicos de que la unidad que sigue es un referente que se presenta como tema de la oración, esto es, constituyen señales léxicas que informan de la existencia de una relación predicativa (en la tradición de estudios ingleses, aboutness) que conlleva la aplicación semántica del predicado a un ente de la realidad extralingüística. A ello debe añadirse el hecho de que estas fórmulas pueden suplir ciertas garantías gramaticales (pronombres correferentes, concordancia o índices 
funcionales), necesarias en otros casos para apuntalar la relación semántica entre tema y comentario.

Así pues, algunas expresiones lingüísticas se han especializado históricamente en introducir un tema en el discurso. Son locuciones como acerca de, en cuanto a, en relación con, con respecto a, por lo que se refiere $a$, etc. ${ }^{1}$ Se trata de fórmulas que presentan un alto grado de fijación, puesto que no admiten variación morfemática (3a) ni la interposición de otras unidades entre los miembros componentes (3b):

$$
\begin{aligned}
& \text { a. *Por los que se refieren a mi hermana...; *En cuantos a mi hermana... } \\
& \text { b. *Con la principal referencia a ... }
\end{aligned}
$$

Algunas de ellas presentan variación libre en las preposiciones inicial y final: por lo que concierne $a \sim$ en lo que concierne a, en relación con $\sim$ con relación a. Estas variantes no se corresponden con otras de contenido, lo que parece indicar un estadio anterior a la fijación definitiva.

En cuanto a su aporte léxico, tiene que ver con los rasgos de las unidades componentes. En algunos casos, aparecen formas verbales o deverbales de verbos dicendi, como referir o hablar (por lo que se refiere a, hablando de). En otros, está implicada la noción semántica de ‘correspondencia' o 'relación’ (por lo que corresponde a, en relación con) , o bien la de ‘tema', ‘asunto’ o 'materia’ (en el tema de, en el asunto de, en materia de).

Sin embargo, a pesar de las similitudes que muestran, no todas estas fórmulas presentan el mismo valor en lo relacionado con la configuración temática del discurso. Una buena parte de ellas introducen temas, o aspectos en los que se desglosa el tema discursivo. Otras, por el contrario, anuncian un cambio de tema o una digresión. Entre las primeras, más abundantes, se encuentran las del tipo en relación con. Uno de sus rasgos más característicos es que no subsisten sin el segmento de categoría sustantiva que expresa el tema de la oración. En el otro extremo se halla por cierto, que nunca se presenta formando grupo con otra unidad. A medio camino entre ambas aparece a propósito, que

\footnotetext{
${ }^{1}$ Una relación pormenorizada (aunque quizá no del todo exhaustiva) puede encontrarse en Fernández Lorences 2006: 235-71).
} 
a veces va seguido de un sustantivo precedido de la preposición de y otras aparece de forma escueta. En lo que sigue, se analizan y contrastan sus diferencias en el plano gramatical, así como el valor que aportan a la estructura informativa del discurso.

2. La fórmula en relación con $(\sim$ en relación a con relación a)

Se trata de una locución formada por PREP. + SUST. + PREP., que aparece siempre seguida de otra unidad de categoría sustantiva que se presenta como tema de la oración. En su composición interna intervienen las preposiciones inicial y final, que en este caso presentan variación libre (con relación a, en relación a, en relación con), y el sustantivo relación (también en lo relativo a). Como señalábamos, ninguna de estas variantes se corresponde con otras de contenido, por lo que cabe suponer que esta locución aún no se ha fijado definitivamente. El sustantivo relación indica «conexión, correspondencia de una cosa con otra», de manera que la unidad que le sigue, presentada como tema, queda marcada como la cosa a la que se está haciendo relación, el asunto sobre el que versa la predicación que sigue.

Podría pensarse que la preposición final cumple la función de subordinar el sustantivo al segmento anterior, pero de hecho no tiene aquí ningún papel como transpositor sino que forma parte de la locución. No hay, pues, un núcleo y un adyacente, y prueba de ello es que el primer componente de la fórmula no tiene autonomía sin el segmento que le sigue, como se evidencia al aplicar la prueba de la conmutación por cero. Ello queda de manifiesto si se contrasta (4a) con (4b): en este último se ha eliminado el tema lingüístico, y ello impide la viabilidad de la construcción:

(4) a. En relación con la información publicada ayer por este periódico titulada «Cascos inaugura una autopista sobre cuya adjudicación a Castellana de Autopistas la UE pidió su anulación por graves irregularidades» [,] el Ministerio de Fomento envió ayer una nota en la que «rectifica dicha información y realiza las siguientes aclaraciones»: [...]. (CREA, La Razón, 09/04/2003)

b. *En relación, el Ministerio de Fomento envió ayer una nota...; *En relación con, el Ministerio de Fomento... // En relación con ello $\{\sim$ a eso $\}$, el Ministerio de Fomento... 
Hay que concluir, pues, que se trata de una locución prepositiva, hecho que se confirma además al comprobar que en el ámbito suboracional puede intervenir como transpositor de un sustantivo a categoría adjetiva, posibilitando que se subordine a otro sustantivo:

Las ideas en relación con sentimientos negativos deben ser eliminadas; La respuesta en relación a la escala queda, momentáneamente, diferida. (CREA, Revista Estadística Española, n. $\left.{ }^{\circ} 149,01-04 / 2002\right)$

Lo que aporta la locución, frente a una preposición léxicamente más transparente como $a$ o de, es el rasgo semántico de ‘relación’ entre una cosa y otra. En algunos contextos, resulta equivalente a otras como sobre o acerca de:

\footnotetext{
Esto nos daría una clave importante en relación a todo lo que hemos dicho acerca del personaje cunillesco: que se aborda desde la pluralidad de personajes y no desde un reduccionismo, digamos, costumbrista o psicologista. (CREA, Stichomythia. Revista de teatro español contemporáneo, $\mathrm{n}^{0} 0,01 / 2002$ )
}

Cuando en la oración aparece un verbo de lengua, la locución puede introducir un grupo sustantivo que, en función de suplemento, configura el tema del discurso referido (Fernández Lorences 2012):

Habló en relación con las nuevas disposiciones, y dijo que los bancos tenían que decidir ahora si se adherían a la normativa europea.

Llegados a este punto, creemos que es importante tener en cuenta la estructura temacomentario en los enunciados bimembres o categóricos, y la posición inicial que en la oración ocupan las unidades tematizadas, puesto que esta configuración sintagmática puede explicar, como se verá más adelante, las diferencias entre unidades muy cercanas en el plano gramatical pero que difieren en cuanto a los valores discursivos relacionados con el tema.

En el caso de la fórmula en relación con, esta actúa como presentadora de un tema cuando aparece en inciso en la posición inicial de la oración (o, en todo caso, antepuesta al predicado); con estas características, puede establecer correlaciones con otras locuciones afines, de modo que el discurso se organiza en secuencias textuales, en bloques informativos cuyo contenido queda marcado por un tema o aspecto del tema, y al que se atienen no solo la predicación de la oración en la que se incluye el inciso temático sino también los comentarios siguientes, al menos hasta que aparezca una nueva marca de tema. Es lo que sucede, por ejemplo, en el texto que sigue: 
(8) En el año 2002 el Gobierno comunitario decidirá sobre la expiración o renovación de tres asuntos importantes. [...]

En cuanto al Coto de Shetland (restringido a las flotas de países del sur, entre ellas la española, porque las especies de estas regiones estaban casi extinguidas) el estudio recoge "que la evolución de las poblaciones no permite ningún aumento del esfuerzo pesquero y considera que deben mantenerse las restricciones".

En relación con el acceso al mar del Norte, también acotado para los buques españoles, la Comisión ratifica que "el acceso a los recursos se limita a las flotas que disponen de cuotas". (CREA, Faro de Vigo, 05/04/2001)

\section{La locución por cierto}

Por cierto constituye también una locución, aunque muestra diferencias notables con la vista anteriormente:

a) En lo que se refiere a las unidades componentes, presenta un mayor grado de fijación, puesto que no admite variantes en la preposición.

b) Respecto de su configuración interna, nunca admite ir seguida por una expresión referencial que exprese el tema.

c) En sus relaciones con la oración en la que se integra, presenta la flexibilidad que caracteriza a los segmentos en función incidental, esto es, puede aparecer como inciso ocupando el primer lugar de la cadena sintagmática, el final de la oración o incrustarse en posiciones intermedias.

d) Con respecto a los rasgos suprasegmentales, se caracteriza por ir seguida de un tonema de cadencia o semicandencia (al contrario de lo que sucede con las fórmulas introductoras de tema, que presentan un ascenso tonal entre el inciso y el resto de la oración).

e) A diferencia de otras fórmulas tematizadoras del tipo con relación a, no conforma una locución prepositiva sino adverbial.

f) Por último, su papel respecto de la estructura temática del discurso no consiste en introducir un tema sino en advertir de una digresión. 
La edición de 1780 del diccionario de la Academia consigna por cierto como expresión adverbial, haciéndola equivalente a ciertamente, a la verdad (NTLLE, s. v. cierto). La definición se mantiene sin cambios hasta la edición de 1983, a la que en este momento se añade un nuevo sentido:

(9) Por cierto. loc. adv. Ciertamente, a la verdad. I loc. conjunt. que introduce un cambio de tema o un paréntesis en el discurso o en la charla: con ella, por regla general, señala el hablante la oportunidad de introducirlos. (NTLLE, RAE 1983, s .v. cierto)

Así que los rasgos léxicos que conlleva, derivados del adjetivo cierto como ‘verdadero', 'seguro', 'indubitable' (DRAE, s. v. cierto) formarían parte de construcciones como la que se registra en este texto de finales del XVI:
Por cierto se tiene que el arzobispado de Sevilla se dará al cardenal de Guevara y la iglesia de Cuenca á Maximiliano de Austria, obispo de Cádiz [...]. (CREA, L. Cabrera de Córdoba (c. 1599-1614), Relación de las cosas sucedidas en la corte de España desde 1599 hasta 1614, Madrid, J. Martín Alegría, 1857)

A partir de la construcción tener (o dar) por cierta una cosa, podría haberse extendido el uso con elipsis del núcleo verbal, dejando paso a la locución seguida de la oración sustantivada, tal como se recoge en el fragmento siguiente:

\begin{abstract}
Sol.- Basta, que todas las que habéis dicho este viaje han sido de alabanzas, y pues se trata desto, os quiero decir de un monasterio que tiene Burgos, que es muy digno della, que, como hombre que no ha estado en él, no le habrá visto, el cual fundó el rey don Alonso otavo de Castilla. Está fuera de la ciudad, es de monjas y se llama Las Huelgas, cuya abadesa tiene debajo de su dominio más de ciento y cincuenta hijas de señores muy principales, y ha habido monjas en él tres infantas doncellas, hijas de grandes reyes de Castilla, las cuales, aunque las traían casamientos para ser reinas, no quisieron serlo. Este monasterio tiene debajo de su juridición otros diez y siete monasterios y trece villas y más de otros cincuenta lugares, y provee doce encomiendas y muchas capellanías, y otros oficios de justicia y regimientos.
\end{abstract}

Rojas.- Por cierto que es notable grandeza, y tanto que parece increíble; y pues llegamos hoy a Burgos temprano, con facilidad podremos ir a vello.

Sol.- Eso y todo lo demás veremos de espacio, que hay mucho que ver en esta ciudad. (CREA, A. Rojas Villadrando (1603): El viaje entretenido, Madrid, EspasaCalpe, 1977).

En este caso, la locución tiene un valor modalizador respecto de la predicación, y vendría a equivaler a la expresión ciertamente. Aunque es necesario un análisis diacrónico más exhaustivo para confirmarlo, podríamos suponer que a partir de usos en que por cierto ocupaba la primera posición de la cadena sintagmática la fórmula pudiera 
haberse ido diversificando y dando lugar a dos construcciones diferenciadas tanto en el plano semántico como sintáctico. Una de ellas, con el valor de modalizador de la oración, se habría ido configurando en la forma ciertamente, dejando el valor digresor para la locución por cierto.

Con todo, el uso como digresor — $-\mathrm{O}$, al menos, como unidad situada en una frontera sutil entre los valores de digresión y modalización- ya está presente en etapas tempranas del castellano, como atestigua el texto de Cervantes:

—Yo iré y volveré presto — dijo Sancho—; y ensanche vuestra merced, señor mío, ese corazoncillo, que le debe de tener agora no mayor que una avellana, y considere que se suele decir que buen corazón quebranta mala ventura, y que donde no hay tocinos, no hay estacas; y también se dice: «Donde no piensa, salta la liebre». Dígolo porque si esta noche no hallamos los palacios o alcázares de mi señora, agora que es de día los pienso hallar, cuando menos los piense; y hallados, déjenme a mí con ella.

- Por cierto, Sancho — dijo don Quijote_- que siempre traes tus refranes tan a pelo de lo que tratamos cuanto me dé Dios mejor ventura en lo que deseo. (CREA, M. de Cervantes Saavedra (1615), El ingenioso hidalgo don Quijote de la Mancha, Barcelona, Instituto Cervantes/Crítica, Barcelona, 1998).

Su rendimiento textual como unidad que permite introducir una digresión en el discurso ha sido señalado en numerosos trabajos lexicográficos y gramaticales ${ }^{2}$ (Moliner 196667, Fuentes Rodríguez 1992, Mateo Rodríguez 1996, Martín Zorraquino y Portolés Lázaro 1999, Acín Villa 2000, Fernández Lorences 2010, entre otros). A la hora de analizar la movilidad que presenta esta unidad, se ha constatado su gran flexibilidad de posición respecto de la oración en que se inserta, aunque esto mismo originaría alguna dificultad para determinar cuándo estamos ante una expresión adverbial integrada en la oración o, por el contrario, ante «un marcador de digresión»:

(13) Aunque fructífera académicamente, su estancia en los EE. UU. no fue por cierto muy agradable. (Mateo Rodríguez 1996: 539)

\footnotetext{
${ }^{2}$ El valor de esta unidad como elemento que advierte de una digresión ha sido recogido en los diccionarios y en los numerosos estudios que se han realizado sobre los llamados «marcadores discursivos». Algunos autores le han dedicado trabajos específicos: Fuentes Rodríguez (1992) se ocupa delas diferencias entre el adjetivo cierto, el adverbio de enunciación ciertamente y los valores en el discurso de por cierto; Mateo Rodríguez (1996), por su parte, relaciona estos últimos con su posición sintagmática, mientras que Acín Villa (2000) los contrasta con los de otros digresores como a propósito y hablando de.
} 
Su posición en la oración no es, en efecto, un rasgo secundario con respecto a sus valores discursivos o a su función sintáctica. El ejemplo recogido en (13) viene a mostrar su carácter adverbial, puesto que ahí solo cabe concluir que aparece integrado en una función suboracional, como adyacente de un adverbio o de un adjetivo, al igual que lo haría el adverbio ciertamente (o cualquier otro en función de término terciario):
a. *Aunque fructífera académicamente, su estancia en los EE. UU. no fue por cierto.
b. Aunque fructífera académicamente, su estancia en los EE. UU. no fue agradable;
c. Aunque fructífera académicamente, su estancia en los EE. UU. no fue muy agradable.
d. Aunque fructífera académicamente, su estancia en los EE. UU. no fue por cierto (ciertamente $\sim$ precisamente, realmente...) muy agradable.

El sentido de digresión que parece aflorar en este caso quizá sea un indicio de que los valores de modalización en ciertamente y de digresión en por cierto no estén aún totalmente separados, o al menos no lo estén en ciertos contextos sintácticos. ${ }^{3}$

Al margen de estos usos, no se discute su papel como digresor cuando se aloja como inciso en la oración, aunque puede plantearse el problema de determinar qué secuencia textual es la que queda marcada como digresiva cuando ocupa otras posiciones que no son la inicial. En este sentido, Mateo Rodríguez (1996: 539 ss.) contrasta textos con la fórmula o sin ella, para concluir que «la presencia o ausencia de marcador confiere, en definitiva, carácter digresivo o pertinente a las secuencias». Sostiene este autor que en algunos contextos la ausencia de la marca obliga al receptor a reponer la relación entre lo dicho anteriormente y lo que sigue, mientras que en otros no cabe sino inferir un cambio de tema.

\footnotetext{
${ }^{3}$ Mateo Rodríguez (1996: 547) hace notar que en algunas ocasiones por cierto admite ir seguido de la conjunción que, con o sin pausa entre ambos, algo que, en su opinión, se debería más a una variación de estilo y, quizá, a una diferencia en el grado de énfasis. Pero de hecho hay dos posiciones en que por cierto no tolera esta combinación. Una de ellas se presenta cuando por cierto se antepone a una oración de modalidad interrogativa (*Por cierto que, ¿a qué hora termina la sesión?); la segunda, cuando la locución se pospone a su foco (Tengo un libro que saldrá en Anagrama el año que viene, un libro muy ilustrado, por cierto (*que). Por su parte, ciertamente se combina también en algunos casos con la conjunción. Parece hacerlo cuando se antepone al predicado al que modifica y no va entre pausas: $E l$ contagio del Báltico ciertamente que aumentará la inquietud en los ucranianos; Y ciertamente que ahí el voto se va a dar con mayor espontaneidad ( $\neq$ Empiezan ya las fiestas taurinas de Madrid y sé ciertamente que no voy a ir a ninguna corrida).
} 
Llegados a este punto, creemos necesario volver sobre lo que se entiende por ‘digresión’. Se ha definido como el «efecto de romper el hilo del discurso y de hablar en él de cosas que no tengan conexión o íntimo enlace con aquello de que se está tratado» (DRAE, s v. digresión), y también el «apartamiento en un relato o exposición del asunto principal, para ocuparse incidentalmente de alguna cosa que surge en relación con él» (DUE, s. v. digresión). Para Martín Zorraquino y Portolés (1999: 4090), los digresores «son estructuradores de la información que introducen un comentario lateral en relación con el tópico principal del discurso».

Ahora bien, en esas definiciones parecen solaparse dos aspectos que pueden revelar valores discursivos ligeramente diferentes y que, además, guardan una estrecha relación con la posición sintagmática de la unidad considerada. De un lado, cabe entender la digresión como el hecho de introducir una información secundaria, un comentario colateral en relación con el tema del discurso. De otro, puede concebirse como una ruptura del hilo discursivo que da entrada a un nuevo tema. Examinamos a continuación algunos ejemplos de uno y otro caso. En (15-18) el digresor no ocupa la primera posición de la oración sino que se incrusta en ella:

(15) La convivencia nos proporcionó grandes momentos: juegos en la playa mezclados con lluvia inesperada (por lo que tuvimos que jugar a la caza del refugio bajo los toldos), teatro sorpresa elaborado por algunos participantes ( $y$ de gran calidad escénica, por cierto) juegos y marujeos bajo el sol en la piscina, reuniones nocturnas en los pasillos y muchos, muchos momentos cargados de risas y aplausos. (CREA, Tercer Sentido 34, 03/2002)

(16) El Ministerio de Educación impulsará nuevas medidas. Entre ellas, la evaluación del desempeño docente y un programa especial de Matemática y Lenguaje. Sin embargo, la calidad no se impone por decreto ni se conquista sólo con dinero. Requiere del esfuerzo y el compromiso de todos: docentes, padres, madres, estudiantes, universidades, alcaldes; también de los medios de comunicación y, por cierto, del Gobierno, que debe crear más conciencia e impulsar una gran campaña nacional para promover a la educación como la gran riqueza de Chile. (CREA, La Tercera, 23/04/2003)

(17) Continuamente estoy trabajando, no paro. Tengo un libro que saldrá en Anagrama el año que viene que se llama «Patologías de la imagen», un libro muy ilustrado, por cierto. Y estoy trabajando en un encargo sobre Valdelomar. (CREA, Revista Telos. Cuadernos de Comunicación, Tecnología y Sociedad, 01-03/2004, nº 58, Segunda Época).

(18) [A]. ¿Entonces, «La edad del silencio» es una apología de la maldad? 
JB. Definitivamente no. Es simplemente el primer intento, por cierto inconcluso, de una disección muy parcial de una conducta vigorosa, de una conducta clara, precisa [...]. (CREA, Ahora, 26/05/2003)

En (15), la locución se sitúa tras un grupo sintagmático (y de gran calidad escénica) configurado como un inciso entonativo, como muestra en el nivel fónico la ruptura de la curva melódica, representada gráficamente por los paréntesis. El inciso aporta una predicación secundaria referida a una unidad integrada en la oración (en este caso, «teatro sorpresa»), y el digresor cumple un doble papel: de un lado, opera como una señal de que lo que se dice se considera secundario respecto del resto; de otro, remite al emisor en cuanto que pone de relieve esa información secundaria. Tal como se ha señalado $^{4}$,

\begin{abstract}
El inciso oracional es [...] un recurso en la configuración semántica del texto, que, en términos generales, no consiste en otra cosa que en una jerarquización de contenidos, en cierto modo al margen de la estructura sintáctica. En la progresión temática, el inciso es siempre una parada (a veces una vuelta atrás) en el desarrollo del argumento narrativo o en la exposición conceptual. (Martínez 2004: 115)
\end{abstract}

Así pues, es el hablante el que establece el rango informativo de sus aportaciones, de manera que ese mismo contenido podría aparecer, en otros casos, en el mismo plano semántico y con una configuración sintáctica que así lo refleje. Una prueba de ello es que el segmento parentético podría yuxtaponerse o coordinarse con el anterior sin que se malograra la coherencia del texto. Se perdería, eso sí, el juego de planos informativos que el emisor pretende transmitir:

(20) La convivencia nos proporcionó grandes momentos: juegos en la playa mezclados con lluvia inesperada (por lo que tuvimos que jugar a la caza del refugio bajo los toldos), teatro sorpresa elaborado por algunos participantes y de gran calidad escénica, juegos y marujeos bajo el sol en la piscina, reuniones nocturnas en los pasillos y muchos, muchos momentos cargados de risas y aplausos.

En (16) sucede algo parecido, solo que por cierto se incrusta en la oración entre dos unidades coordinadas (los medios de comunicación y el Gobierno). Antepuesto a la última, la destaca, de modo que su eliminación supone una secuencia formalmente aceptable pero en la que se pierde el relieve de la unidad antes realzada:

\footnotetext{
${ }^{4}$ El análisis de Martínez (2004) se centra expresamente en las oraciones inciso independientes, dejando a un lado las subordinadas y otros incisos nominales. Con todo, lo que se sostiene respecto de estos segmentos parentéticos resulta aplicable, en nuestra opinión, al comportamiento semántico del digresor.
} 
(21) Sin embargo, la calidad no se impone por decreto ni se conquista sólo con dinero. Requiere del esfuerzo y el compromiso de todos: docentes, padres, madres, estudiantes, universidades, alcaldes; también de los medios de comunicación y del Gobierno, que debe crear más conciencia e impulsar una gran campaña nacional para promover a la educación como la gran riqueza de Chile.

En los ejemplos de (17) y (18), reproducidos más abajo como (22a) y (23a), podemos constatar cómo esta unidad puede anteponerse o posponerse a otro segmento, que queda de este modo destacado ${ }^{5}$ aun cuando se presente como información colateral. Por el contrario, su ausencia hace que esa llamada de atención sobre una parte del enunciado desaparezca (22b y 23b):

(22) a. Tengo un libro que saldrá en Anagrama el año que viene que se llama «Patologías de la imagen», un libro muy ilustrado, por cierto.

b. Tengo un libro que saldrá en Anagrama el año que viene que se llama «Patologías de la imagen», un libro muy ilustrado.

a. - ¿Entonces, «La edad del silencio» es una apología de la maldad? Definitivamente no. Es simplemente el primer intento, por cierto inconcluso, de una disección muy parcial de una conducta vigorosa, de una conducta clara, precisa [...].

b. _ ¿Entonces, «La edad del silencio» es una apología de la maldad? Definitivamente no. Es simplemente el primer intento, inconcluso, de una disección muy parcial de una conducta vigorosa, de una conducta clara, precisa [...].

Si en estos casos la locuación parece estar al servicio de introducir una información que se presenta como secundaria y a la que dota de cierto relieve, cuando la unidad ocupa el primer lugar de la oración advierte inequívocamente de que la predicación que sigue no guarda una relación directa con el tema del discurso. Es lo que sucede en ejemplos como los siguientes:

(24) Carlos, ya puedes seleccionar siete o ocho chicos porque dentro de un momento vamos a hacer una ronda para que cada uno de ellos nos seleccione dos palabras que les guste, dos palabras que les parezcan ricas, que les parezcan llenas de contenido. Por cierto, director, que nos llaman algunos oyentes que quieren saber si se dice espúreo o espurio. (CREA, «Hoy por hoy», 24/04/99, Cadena SER 3/3)

(25) Y y está el señor Lázaro Carreter con nosotros. Dentro de un momento dialogaremos un poco con él. Ahora de nuevo «El lector por horas». Por cierto, quiero decir a los oyentes que nos llamaron con dudas que, espero, el profesor Lázaro Carreter dentro

\footnotetext{
${ }^{5}$ Mateo Rodríguez (1996: 549-550) hace notar su valor como elemento enfatizador cuando aparece en determinadas posiciones de la oración.
} 
de un momento puede pues disipar algunas de ellas porque, en efecto, estamos recibiendo muchas llamadas. Nos encanta saber que la lengua española es querida y es amada y que hay preocupación por cuidarla mimosamente. Bien, seguimos, «El lector por horas». Ahora de La vida es sueño, de Calderón. Sueña el rico en su riqueza que más cuidados le ofrece, sueña el pobre que padece su miseria y su pobreza, sueña el que a medrar empieza, sueña el que afana y pretende, sueña el que agravia y ofende, y en el mundo, en conclusión, todos sueñan lo que son, aunque ninguno lo entiende. Yo sueño que estoy aquí de estas prisiones cargado y soñé que en otro estado más lisonjero me vi. (CREA, «Hoy por hoy», 24/04/99, Cadena SER 3/3)

En (24), la aparición de por cierto supone una ruptura del hilo discursivo y un cambio de tema: se está hablando de hacer una ronda para seleccionar algunas palabras, y se interrumpe la cuestión para referirse a las dudas que manifiestan los oyentes. En (25) el locutor la utiliza para introducir otro asunto, las llamadas de los oyentes; la digresión se cierra más adelante con la expresión bien, seguimos.

Nótese, por otra parte, que en estos casos por cierto admite ser sustituido por la forma $a$ propósito (26). Esta sustitución resulta mucho más difícil cuando la locución focaliza una unidad situada en el interior de la oración (27):

Carlos, ya puedes seleccionar siete o ocho chicos porque dentro de un momento vamos a hacer una ronda para que cada uno de ellos nos seleccione dos palabras que les guste, dos palabras que les parezcan ricas, que les parezcan llenas de contenido. Por cierto ( $\sim$ A propósito), director, que nos llaman algunos oyentes que quieren saber si se dice espúreo o espurio; Ahora de nuevo El lector por horas. Por cierto ( A propósito), quiero decir a los oyentes que nos llamaron con dudas que, espero, el profesor Lázaro Carreter dentro de un momento puede pues disipar algunas de ellas.

... juegos en la playa mezclados con lluvia inesperada (por lo que tuvimos que jugar a la caza del refugio bajo los toldos), teatro sorpresa elaborado por algunos participantes (y de gran calidad escénica, por cierto [\#a propósito]) juegos y marujeos bajo el sol en la piscina...; Requiere del esfuerzo y el compromiso de todos: docentes, padres, madres, estudiantes, universidades, alcaldes; también de los medios de comunicación y, por cierto [\#a propósito], del Gobierno, que debe crear más conciencia...

De lo anterior se desprende que si por cierto se sitúa antes del predicado advierte de una digresión respecto del tema, mientras que si aparece incrustado en la oración lo hace para refererirse a determinada unidad que, en una función oracional, suboracional o extrapredicativa, aporta una información que se presenta como secundaria y a la que la locución, por su mera presencia, destaca. 


\section{La fórmula a propósito}

El papel como digresor de a propósito ha sido señalado en estudios anteriores (Casado 1993, Mateo Rodríguez 1996, Martín Zorraquino y Portolés Lázaro 1999, Acín Villa 2000, Fernández Lorences 2010, entre otros). Se trata de una locución en la que interviene el sustantivo propósito, que al margen de ella puede desempeñar una función oracional en la que mantiene el significado primario de 'intención', tal como se recoge en el ejemplo de Moliner (DUE, s. v. propósito):

(28) Tiene el propósito de marcharse al extranjero.

El sustantivo, precedido de la preposición $a$, forma una locución de carácter adverbial equivalente en su sentido a expresamente, y en la que subsiste el rasgo de 'intención’:

(29) No lo hizo a propósito.

Ya en la edición de 1737 la Academia recogía para esta fórmula el significado de

(30) «Modo adverb. con que se expressa, que alguna cosa es proporcionada ù oportúna para el fin que se deséa ò à que se destina. Por ser el Santo natural de su Obispado, y tan vecino à Osma, como es Caleruega, venía mui à propósito para reformación que pretendía en su Iglesia. (NTLLE, RAE 1737)

En esa misma edición ya se recogen dos variantes formales que se corresponden con otras de significado: a propósito ('oportuno para algún fin’) y de propósito ('con intención, voluntariamente'). Como se observa en el ejemplo anterior en el que interviene el verbo venir, este pierde su valor como verbo de movimiento, pasando a designar, junto con la locución, la idea de 'resultar adecuada una cosa para un fin’.

La Academia da cuenta también de la frase fuera de propósito:

Fuera de propósito. Phrase adverb. con que se expresa, que alguna cosa no viene al caso, ò que es fuera de tiempo ò oportunidad. (NTLLE, RAE 1737)

La idea de ‘adecuación’ persiste en los usos actuales cuando se hace referencia a un acto de habla, en expresiones que informan sobre la pertinencia (o falta de relevancia) de las contribuciones al tema discursivo: Eso no viene al caso, o Eso no viene a propósito para lo que estábamos hablando. 
Como fórmula fijada, compuesta de la preposición a más el sustantivo propósito, pervive en el español actual con comportamientos sintácticos y valores semánticos diferentes. $^{6}$

En primer lugar, subsiste como expresión de carácter adverbial con significado de ‘intencionadamente’, subordinándose al núcleo verbal en las funciones de aditamento o predicativo:

Habíamos quedado a las siete y llegó tarde a propósito.

En segundo lugar, configura una locución de carácter preposicional que permite que un sustantivo o grupo sustantivado pueda subordinarse al verbo como aditamento:

Pero no sólo la seguridad ciudadana está en peligro bajo el Gobierno del PP. Las políticas del PP han generado inseguridad en el trabajo, como evidencian las escalofriantes cifras de siniestralidad laboral; inseguridad alimentaria, como se pudo comprobar con el caso de las «vacas locas»; inseguridad medioambiental, como desgraciadamente estamos sufriendo a propósito de la catástrofe del «Prestige»; inseguridad vial, como tristemente podemos comprobar cada fin de semana en las carreteras españolas; e inseguridad a través de la manipulación informativa de los medios de comunicación. (CREA, El Socialista, nº 651, 04/2003)

(34) En Cataluña, el intento de reconstruir el ejército tras la pérdida de Tarragona, aunque cuenta con el apoyo de la Junta Superior, ocasiona roces y protestas a propósito de la conducta de unos militares que apalean y apresan a varias autoridades locales en Casserras y en La Bisbal [...]. (CREA, Hispania Nova, nº 3, 2003)

Cuando interviene un verbo de lengua, la locución se hace equivalente a otras preposiciones o locuciones prepositivas (sobre, acerca de) que suelen presentar el tema del discurso referido (Fernández Lorences 2012), aquello sobre el que se dice algo, y lo hace a través de una construcción suplementaria:

Antonio Sánchez Barbudo dice a propósito de esa revista: «Aunque sus páginas no aparezcan amarillentas o rosadas por los años ni sus lomos exhalen esa humedad nostálgica que nos habla del frescor y la intimidad de otro tiempo, allí esperábamos hallar en esta larresca edición de El Pasajero una violeta olvidada o el esqueleto de

\footnotetext{
${ }^{6}$ Resulta curioso constatar la evolución histórica de esta forma (tal como me hizo notar el prof. José Polo, a quien agradezco sus certeras observaciones), que podría esquematizarse de este modo: SUST > ADV $($ PREP + SUST) $>$ SUST. Efectivamente, un primitivo sustantivo (propósito) da lugar a un adverbio al unirse a una preposición (a propósito). Y este adverbio, a su vez, origina un nuevo sustantivo (apropósito): «Pieza teatral breve, de circunstancias y de carácter cómico» (DEA, s. v. apropósito).
} 
cualquier flor silvestre. La lectura de estas páginas, ingeniosas, apasionadas, nos deja también en la mente la sugestión de una silueta romántica: el desterrado». (CREA, Espéculo, 06/2003)

Así que en unos casos adquiere el sentido de 'causa', mientras que en otros presenta el de 'tema', aunque siempre subsiste la idea de relación entre una cosa y otra. Una diferencia notable que permite separar estos valores es la posibilidad de que la locución admita ser sustituida por sobre o acerca de, algo que sucede en (36) pero no en (37):

Recordemos lo que comentaba Nora Ephron en Ensalada loca (1975) a propósito de [ sobre, acerca de] los grupos de concienciación. Estos grupos tenían como objetivo «desarrollar la sensibilidad personal hacia los diversos niveles y formas que adopta la opresión en nuestras vidas diarias», «demoler en nuestras mentes la barrera entre lo privado y lo público». (CREA, C. Alborch, Malas. Rivalidad y complicidad entre mujeres, Madrid, Agilar, 2002).

(37) Y hoy ha llegado en ese plan y, a propósito de [\#sobre, acerca de] la cena de los niños o algo por el estilo, ha pasado de las palabras a las obras, le ha dado dos bofetadas y se ha marchado luego y aún no ha vuelto. Ésa es toda la desagradable historia. (CREA, G. Salvador Caja, El eje del compás, Barcelona, Planeta, 2002)

En relación con el tema del discurso, la locución opera en dos sentidos: de un lado, puede presentar el tema discursivo; de otro, puede advertir de una digresión. Cuando se trata de presentar el tema, exige ocupar la primera posición e ir seguido de un sustantivo o unidad sustantiva que codifica el asunto o materia de que trata el texto. Con ese mismo valor de presentador temático, puede aparecer formando parte de un título que, generalmente, constituye una frase nominal; aquí resulta también intercambiable con otras preposiciones como sobre o acerca de:

A propósito de una biografía del escritor británico Samuel Pepys

Autor del más famoso diario escrito en inglés, Samuel Pepys (1633-1703) vivió algunos de los acontecimientos más relevantes de la historia de Inglaterra del siglo XVII: la guerra civil que resultó en la victoria de Cromwell y la decapitación del malhadado Carlos I (1649); la restauración de la monarquía en 1660; la terrible peste de 1665, y el gran incendio de Londres al año siguiente. Durante la mayor parte del periodo de 1660 a 1689, Pepys desempeñó el cargo de secretario del ministerio de la marina, en que dio prueba de sus dotes geniales de administrador civil. Trabajador incansable, inteligente, meticuloso, si bien capaz de rebajarse a corruptelas y sobornos, llevó a cabo, por sus propios esfuerzos, la reforma de la administración naval. (CREA, El País, Babelia, 2/03/2003)

Con el valor de advertir de una digresión, puede presentarse la expresión escueta (3941) o bien ir seguida de un sustantivo (42-43): 
(39) Hay fórmulas simples, y técnicamente viables, como que quienes lo soliciten tengan un servicio sin proxy (o inversamente: que sólo tengan proxy quienes lo pidan). La empresa responsable debería permitir esta opción, y en general no establecer modificaciones tan críticas sin un debate público con sus usuarios. A propósito: estando el sector de las telecomunicaciones tan regulado como está, y dado que un servicio vital como el del acceso a Internet está en manos de un monopolio de facto, quienes velan por el bien común no deberían permitir que ocurrieran cosas como éstas, que minan la misma base sobre la que los españoles pueden ganar en innovación y experiencia en un terreno básico para nuestro futuro. (CREA, El País, Ciberpais, 30/01/2003)

(40) Y... habiendo sido director, ponerme a hacer crítica... tampoco era crítica, era una sección de información en la revista Claudia. A propósito, recuerdo de haber puesto algo que había descubierto en una publicación de afuera. Leí que los japoneses estaban experimentando con cine en cinta, lo que ahora se llama video. Y me acuerdo haber escrito que eso era una maravilla, etc., etc. (CREA, Film [on line], 06-07/2003)

(41) Si se fija usted, en la existencia humana ocurre algo paradójico: cuanto más se necesita, se desea y se busca, por ejemplo, la aprobación, la seguridad, la felicidad, la salud, el éxito...más difícil resulta conseguir lo que ansía. Y al revés: cuanto menos se preocupa uno de esas cosas -simplemente se ocupa- más fácilmente las logra.

A propósito, ¿quién no ha leído o escuchado alguna vez la fábula del gato que pretendía ser feliz con sólo cazar su cola? (CREA, Eco Católico, 11/05/2003)

Cuando va seguida de un sustantivo, este representa la entidad que al ser mencionada ha suscitado una idea paralela o secundaria; en todo caso, la expresión tiene como función advertir al interlocutor de que la predicación siguiente rompe el hilo discursivo o, en todo caso, no supone una contribución relevante al tema:

(42) Tiene razón la baronesa de Dumbría, al llamarme el de la mano horadada. Razón: y sin embargo, me ataca los nervios al darme consejos de economía; es como si a una adelfa la dijesen: «Maldita, sé garbanzo, que te conviene mucho.»

A propósito de garbanzos: mi comida es una desolación, y apenas digiero. Ando a salto de mata, hoy en un bodegón, mañana en Fornos; me desayuno con salchichón o queso [...]. (CREA, E. Pardo Bazán, La Quimera, Madrid, Cátedra, 1991).

(43) - -Te peinaré primero... Estate quieto... No hagas visajes...

— ¡Oh, no seas cruel!... ¡Qué suplicio!

—Si no es nada, hijito... Quieto. Déjame sacar bien la raya. Apenas es importante la raya.

— A propósito de raya... ¿Qué es eso del límite que dijo Cruz? No he pensado en otra cosa durante toda la noche. ¿Quiere decir que hemos llegado al límite de nuestro sufrimiento? 
-Sí. (CREA, B. Pérez Galdós, Torquemada en la cruz, Biblioteca Virtual Miguel de Cervantes, Alicante, Universidad de Alicante, 2002)

La relación entre lo mencionado y lo que sigue puede ser inconsistente, como muestran los ejemplos anteriores. El valor que tiene la locución no es tanto presentar o hacer evidente esa supuesta relación como advertir al interlocutor de que se va a producir un cambio de tema y evitar, por tanto, que la información que se añade se interprete como referida a lo que se ha dicho antes. Así, al tiempo que advierte de la digresión, la fórmula anula el supuesto de que se está incumpliendo el principio de cooperación (Grice 1975) y garantiza la coherencia discursiva.

Bibliografía

Acín VillA, E. (2000): «Por cierto, a propósito y otros digresores», en P. Carbonero Cano, M. Casado Velarde y P. Gómez Manzano (eds.), Lengua y discurso. Estudios dedicados al profesor Vidal Lamíquiz, Madrid: Arco/Libros, págs. 5972.

Álvarez MEnÉNDEZ, A. I. (1993): «Transpositores complejos, conjunciones/preposiciones “impropias” y otras fórmulas alternativas en la introducción de las subordinadas adverbiales», Lingüística española actual XV/1. Madrid: Arco/Libros, 109-148.

Casado Velarde, M. (1993): Introducción a la gramática del texto del español, Madrid: Arco/Libros.

Cifuentes HonRubia, J. L. (2003): Locuciones prepositivas. Sobre la gramaticalización preposicional en español. Alicante: Universidad de Alicante.

HJElmslev, L. (1943): Prolegómenos a una teoría del lenguaje. Madrid: Gredos,1974² (vers. esp. J. L. Díaz de Liaño).

FERNÁNDEZ FERNÁNDEZ, A. (1993): La función incidental en español, Oviedo: Universidad de Oviedo. 
FERNÁNDEZ LoRENCES, T. (2010): Gramática de la tematización en español, Oviedo: Universidad de Oviedo.

FERNÁNDEZ LORENCES, T. (2012): «Hablar y decir: la especialización informativa de los verbos de lengua en español», Verba 39, págs.189-217.

FueNTES RoDRÍGUEZ, C. (1992): «Las coordenadas del discurso: cierto y sus derivados», en Investigaciones semióticas IV (Actas del IV Simposio Internacional de la Asociación Española de Semiótica), vol. II, Madrid, Visor, págs. 897-907.

GRICE, H. P. (1975): «Logic and conversation», en P. Cole y J. L. Morgan (eds.), Syntax and semantics, vol. III: Speech acts, New York, Academic Press, págs. 41-58.

Martín Zorraquino, M. A. y J. Portolés LÁzAro (1999): «Los marcadores del discurso», en I. Bosque y V. Demonte, Gramática Descriptiva de la Lengua Española, Madrid, Espasa, págs. 4051-4213.

MARTíneZ, J. A. (2004): «Algunas oraciones 'parentéticas’ independientes en español», en M. A. Álvarez Martínez y M. S. Villarrubia Zúñiga (eds.), Actas del II Congreso Internacional de la Asociación Coreana de Hispanistas (28 al 30 de junio de 2002), Madrid: Universidad de Alcalá, págs. 113-27.

MARTínez, J. A. (1994a): Propuesta de gramática funcional. Madrid: Istmo.

MARTínEZ, J. A. (1994b): Funciones, categorías y transposición. Madrid: Istmo.

MARTínEZ, J. A. (1994c): Cuestiones marginadas de gramática española. Madrid: Istmo.

Martínez, J. A., A. I. Álvarez Menéndez, Á. Arias Cabal, T. Fernández Lorences, F. Fernández de Castro, A. Fernández Fernández, S. García García, H. Martínez García, A. J. Meilán García y J. San Julián Solana (en prensa): «Léxico, sintaxis y semántica de algunos transpositores complejos».

Mateo Rodríguez, J. E. (1996): «Los marcadores digresivos. Estudio especial de por cierto en español actual», en M. Casado Velarde, A. Freire Llamas, J. E. López Pereira y J. I. Pérez Pascual (eds.), Scripta Philologica in memoriam Manuel Taboada Cid, A Coruña: Servicio de Publicaciones, Universidade da Coruña, t. II, págs. 531-52.

Moliner, M. (1966-67): Diccionario de uso del español. Madrid: Gredos, 1994 (abrev. $D U E)$ 
PAvón Lucero, M. V. (1995): «Clases de partículas: preposición, conjunción y adverbio». En: I. Bosque y V. Demonte (dirs.), Gramática Descriptiva de la Lengua Española. Madrid: Espasa Calpe, 565-653.

Porroche Ballesteros, M. (2006): «Sobre los adverbios enunciativos españoles. Caracterizacion, clasificación y funciones pragmáticas y discursivas fundamentales», Revista Española de Lingüística, 35,2, págs. 495-522.

PoRTOLÉs, J. (1993): «La distinción entre los conectores y otros marcadorees del discurso en español», Verba 20, págs. 141-170.

PORTOLÉs, J. (1998): Marcadores del discurso, Barcelona: Ariel.

Real ACADEMia EsPaÑola (2001): Diccionario de la lengua española. Madrid: Espasa Calpe (22 a ed.) (abrev. DRAE).

REAl ACADEMIa ESPAÑOla: Nuevo tesoro lexicográfico de la lengua española (NTLLE) [en línea]. http://www.rae.es [01/05/2012 - 01/06/2012]

ReAl ACADEMia Española: Banco de datos (CREA) [en línea]. Corpus de referencia del español actual. http://www.rae.es [01/05/2012 - 01/06/2012]

Real Academia Española: Banco de datos (CORDE) [en línea]. Corpus diacrónico del español. http://www.rae.es [01/05/2012 - 01/06/2012].

SECO, M., O. ANDrÉS y G. RAMOS (1999): Diccionario del español actual [abrev. DEA], Madrid, Santillana, 2 vols.

Recibido:14 junio 2013

Aceptado: 15 septiembre 2013

Revisado: 27 octubre 3013

Publicado: 31 octubre 2013 